\title{
Fatal cerebral hemorrhage due to autonomic dysreflexia in a tetraplegic patient: case report and review
}

\author{
I Eltorai MD, R Kim MD, M Vulpe MD, H Kasravi MD, W Ho MD \\ Veterans Affairs Medical Center, Long Beach, California and the Department of Surgery, \\ Pathology and Neurology, the University of California College of Medicine, Irvine, \\ California, USA.
}

Autonomic dysreflexia is the most important specific complication of high level spinal cord injury both in tetraplegic and in paraplegic patients above the midthoracic neural segment. It is a life threatening emergency that may lead to apoplexy. We present a case of fatal cerebral hemorrhage due to autonomic dysreflexia in order to demonstrate the gravity of this particular syndrome.

Key words: autonomic dysreflexia; spinal cord injury; cerebral hemorrhage.

\section{Definition}

Autonomic dysreflexia (AD) is defined as a life threatening emergency that not uncommonly occurs after spinal cord injury (SCI) at the T6 level or above and is manifested by a special clinical syndrome.

\section{Clinical picture}

The most devastating phenomenon is paroxysmal hypertension. The sharp rise of blood pressure (BP) is both systolic and diastolic, and the typical syndrome includes both subjective and objective findings. The former includes pounding headache which may be preceded by abdominal discomfort, especially in the suprapubic region or in the penis, followed by undefined ascending sensation in the midline of the body or a feeling of chest oppression with difficulty in breathing, with shivering or a hot feeling. The pounding headache is noticed particularly in the back of the head, between the eyes, ${ }^{1}$ or bitemporally. There is vasodilation above the level of injury manifested by flushing of the face, neck, upper chest, upper arms and congestion of the nasopharyngeal mucosa leading to stuffiness of the nose (known as Guttmann's sign). This is accompanied by sweating and piloerection (cutis anserina) or goose skin.

Other symptoms are: nausea, dyspnoea and visual disturbances. Although this is a typical picture of the syndrome, in some patients it may be atypical, manifested by only one or more of the above features. The picture may also be chronic; ${ }^{2}$ there can also be an exacerbation of the chronic condition.

Subjective clinical findings are: acute distress, anxiety, apprehension, restlessness, but sometimes none of these symptoms. Above the lesion there is flushed sweating, sometimes excessive. The eyes are congested and the pupils may be dilated. The characteristic signs are mainly cardiovascular: hypertension both systolic and diastolic, bradycardia especially with the high thoracic lesions, and sometimes tachycardia in those with cervical lesions. ${ }^{3}$ Dysrhythmia, especially extrasystoles may be noticeable. Vasoconstriction in the lower extremities both arterial and venous with decrease in the calf blood flow occurs, possibly also in the hands. Neurological changes may be detected especially in severe cases, eg aphasia, blindness, seizures and coma, the latter usually due to intracranial hemorrhage. The acute condition usually subsides with active therapy but in a few cases after resolution and removal of the cause, the patient is prone to recurrent attacks induced by the least stimulus for 7-10 days, a condition termed status dysreflexia by A Graham Smith and quoted by Linden et al.$^{4}$ Hypertension may be the only manifestation 
especially during cystoscopy (Khonsari), and termed by one of us (IE) 'dysreflexia sine cephalalgia'. The management of $\mathrm{AD}$ is well documented in the literature. ${ }^{5-9}$

According to Comarr, ${ }^{2}$ before the recognition of this syndrome, such patients were diagnosed as epileptics or as developing a cerebrovascular accident (CVA) secondary to hypertension. Although AD was described by Hilton, ${ }^{10}$ it was not until 1947 that Guttmann and Whitteridge described the classical cardiovascular picture. The syndrome is now well recognized in the field of SCI and its management is fairly well established.

Hypertension is a leading risk factor for cerebral hemorrhage. In massive intracranial hemorrhage, a history of hypertension was obtained in $94 \% .{ }^{11}$ The most common sites of bleeding are the putamen $(35-50 \%)$, subcortical $(30 \%)$, cerebellar $(16 \%)$, thalamus $(10 \%)$, and pons $(5 \%)$. These figures differ in different series. In national statistics nothing is mentioned about autonomic dysreflexia as a cause of CVA.

To investigate $\mathrm{AD}$ and CVA the senior author sent questionnaires to the directors of Veterans Affairs Spinal Cord Injury Centers (20) and to the Department of Health and Human Services Regional Centers (14). There were 17 responses $(50 \%)$. There were 14 cases of known cerebral hemorrhage. The population of the SCI Centers amounts to about $36,000,11,000$ in the Department of Health and Human Services and 25,000 in the Veterans Affairs System. These cases occurred over many years. The incidence cannot therefore be predicted. Thirteen of the patients had C5-6 tetraplegia and one was a T3 paraplegic. All were males except for one female who was afflicted during cesarean section. All were in the fourth decade of life except for one patient who was in the sixth decade. The outcome was survival with an additional neural deficit, with the exception or one patient who died. According to the National Spinal Cord Injury Data Bank, in Birmingham, Alabama, there were 22 deaths due to CVA amongst 796 deaths (De Vivo, personal communication); this included both hemorrhage and infarctions and there is no mention of $\mathrm{AD}$ as a cause. In this paper a patient with typical autonomic dysreflexia complicated by a fatal cerebral hemorrhage is described.

\section{Case report}

This 36 year old man, a C6 incomplete quadriplegic due to a motor vehicle accident in November 1974, was admitted to the Long Beach Veterans Administration Medical Center on May 15, 1987 for management of bilateral ischial and sacral decubiti that had been present for approximately 6 months. He also claimed a 40 pound weight loss during this period. Past medical history included laminectomy at the time of the injury, recurrent urinary tract infections, bouts of hepatitis B and narcotics addiction, surgery for treatment of pressure sores (most recently $1 \frac{1}{2}$ years prior to admission), progressive loss of function in the left arm leading to a diagnosis of syrinx (by MRI) at another VAMC in January 1987, and persistent esophagitis necessitating insertion of a feeding gastrostomy in March 1987. Physical examination revealed a poorly nourished man with normal vital signs (blood pressure 90/58), ischial and sacral bedsores, a gastrostomy tube and a Foley catheter in place, flexion contractures of the arms and legs, and a C6 sensory level to pinprick.

He received nutritional support (through gastrostomy tube feedings), decubitus care and physiotherapy, and remained stable except for intermittent low grade fever, recurrent urinary tract infections, and persistent diarrhea. Stool cultures bred C. difficile on June 24, 1987, and metronidazole therapy was started. Cystogram on August 17, 1987 revealed the presence of urinary bladder stones, for which he underwent cystolitholapaxy on November 11, 1987. By November 5 his hip decubiti had healed, but not the sacral ulcer. Esophagoscopy on December 1 disclosed the presence of a tight stricture at $40 \mathrm{~cm}$. Dilation was performed on January 5, 1988.

On the evening of January 7 at $10 \mathrm{pm}$ he complained of severe headache, and his blood pressure was noted to be $240 / 120$. The Foley catheter was changed because of drainage problems, and $600 \mathrm{cc}$ of residual 
urine were removed. Shortly thereafter the blood pressure fell to $110 / 60$, and he no longer complained of headache. At 6 am on January 8 his abdomen again appeared to be distended possibly due to fecal impaction; he was recatheterized, but no urine was obtained. Shortly thereafter $(6.10 \mathrm{am})$ the patient was found unresponsive with a blood pressure of $180 / 90$ and pulse rate of 102 ; the pupils were dilated and unreactive. By 7 am, though his blood pressure had fallen to $90 / 70$, his neurological state remained unchanged. At 7.10 am he became apnoeic his pulse was weak, and his blood pressure was unobtainable. He was immediately intubated and ventilated but was completely unresponsive with fixed dilated pupils. CT scan of the head was performed and showed a massive right cerebral hemorrhage with rupture into the lateral, third and fourth ventricles (Fig 1). EEG was isoelectric. Cardiac arrest occurred on January 11, 1988.

Postmortem studies showed a massive right cerebral hemorrhage with rupture into the lateral, third and fourth ventricles with evidence of moderately severe bilateral uncal, transtentorial and cerebellar tonsillar herniation (Fig 2). No aneurysm was seen.

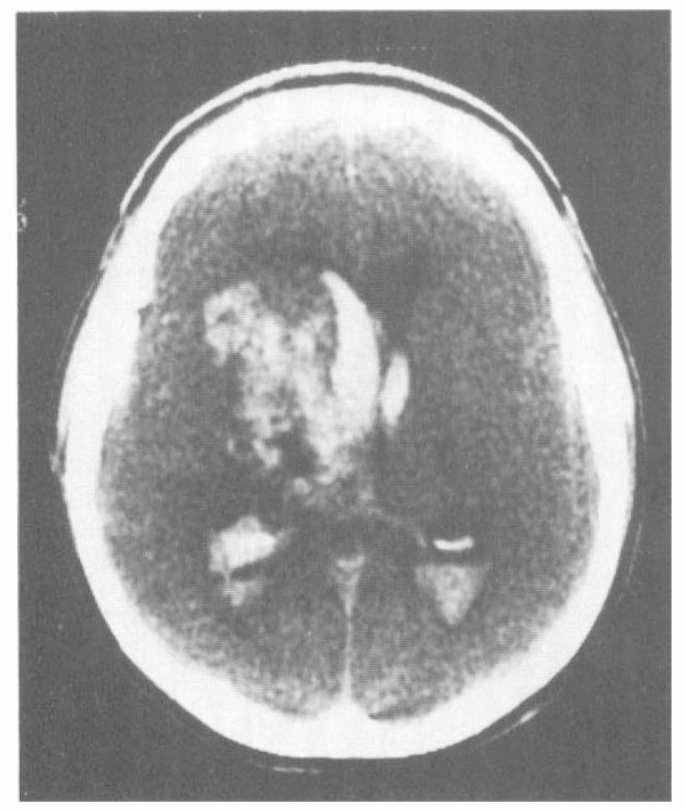

Figure $1 \mathrm{CT}$ Scan showing intracerebral hemorrhage.
The spinal cord showed extensive hemorrhage, traumatic myelopathy of the midcervical region, and upper thoracic syringomyelia. There was no evidence of vascular malformation. The heart showed right and left ventricular hypertrophy, mild right ventricular dilatation and mild atherosclerosis of the coronary arteries.

\section{Discussion}

Autonomic dysreflexia is a serious syndrome that may occur in the spinal cord injured: high paraplegics and tetraplegics. It was recognized only after World War II and its proper management received attention mostly in the last 2 decades. It is the most important specific complication in the spinal cord injured. Although management is simple, serious complications can develop. Management has been established by many authors in the spinal cord injury field.

In our center energetic and aggressive care is very important to avoid serious complications. The physician and the nurse rush to the patient's bedside, the latter raises the head of the bed to the sitting position while the physician conducts a rapid physical examination including the cranial nerves, the abdomen, scrotum and the extremities. During that examination the nurse is monitoring the blood pressure. If sitting up does not start lowering the pressure within a few minutes, one $10 \mathrm{mgm}$ capsule of Nifedipine is given to the patient to crush and swallow. Catheter drainage is checked and treated very gently by small volume irrigation or by change. If there is bowel impaction Xylocane gel $2 \%$ is introduced into the rectum and no rectal evacuation is allowed until the crisis is over. Nifedipine may be repeated in 15 minutes if the hypertension persists. Continuous monitoring every 5-10 minutes is necessary until the blood pressure is stable, watching for hypotension and for rebound hypertension. For persistent hypertension an intravenous infusion is started and the patient should be sent to an intensive care unit for more aggressive measures. The underlying etiologic factor must be corrected to prevent these episodes. Other preventive measures such as nitroglycerine paste; Clonidine patch 


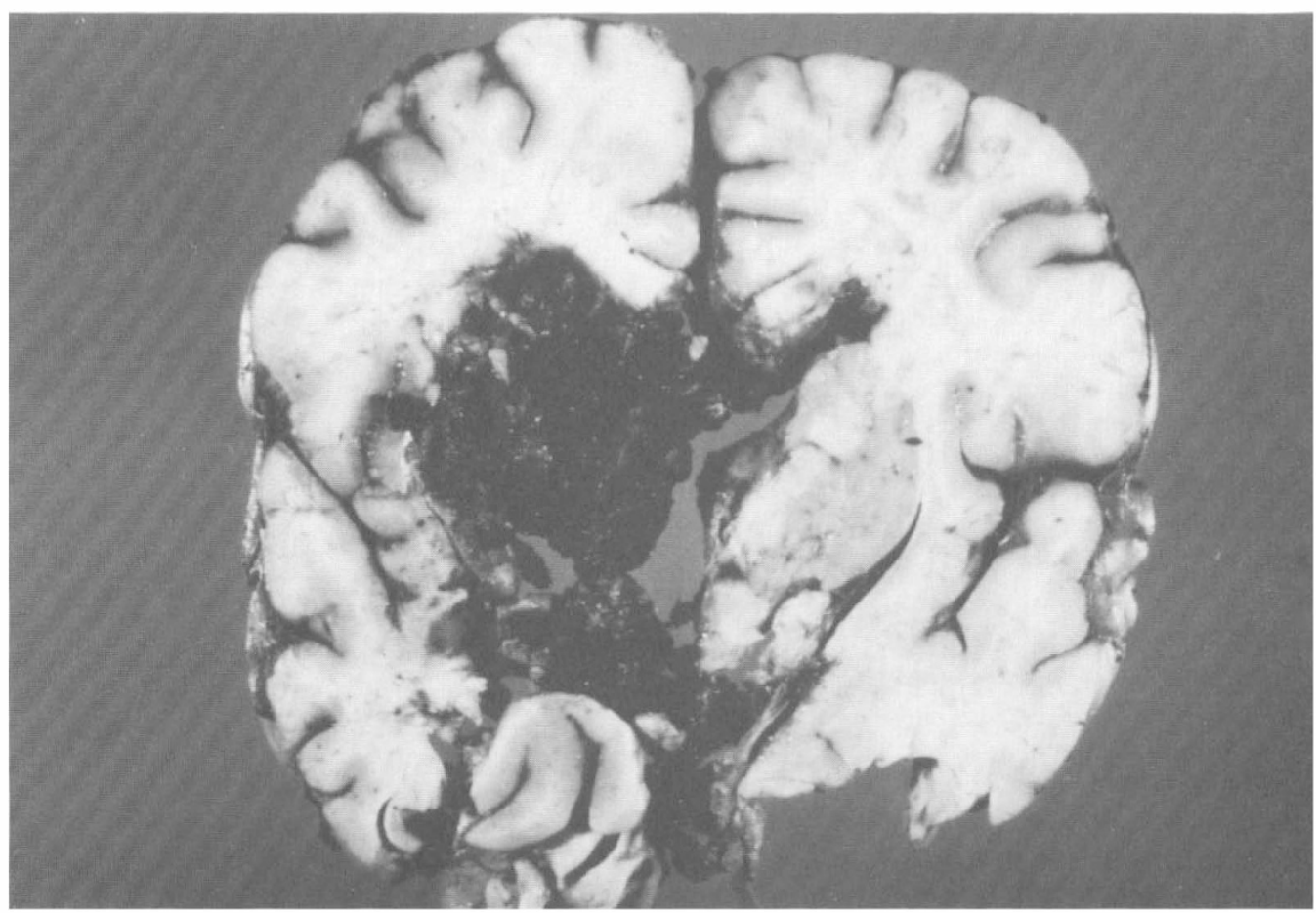

Figure 2 Postmortem gross anatomy of the cerebral hemorrhage.

(Catapress TTS by Boeringer Ingelheim Corp. of Ridgefield, CT 06877); Nifedepine; Apresoline (hydralazine hydrocloride USP, CIBA-GEIGY Corp., Summit, NJ 07901); Labetalol; intravesical pontocaine; or Ditropan (oxybutynin chloride by Marion Laboratories, Inc., Kansas City, MO 64137) may be prescribed.

The case presented here is a typical example of the gravity of AD both in the male and female population; in the latter it can be fatal during delivery. Yarkony et al ${ }^{12}$ collected 10 cases of seizures secondary to autonomic dysreflexia and added 3 of their own independent of cerebral pathology. Guttmann $^{1}$ recorded a female paraplegic who developed seizures with hemiplegia during delivery. Linden et $a l^{13}$ reported a fatal case due to status epilepticus from AD. Kursh et $a^{14}$ reported a case of fatal intracerebral hemorrhage during catheterization. Another patient of the same authors developed seizures with aphasia due to subarachnoid hemorrhage but eventually recovered. Hanowell and Wilmot ${ }^{15}$ re- ported a case of $\mathrm{C} 4$ incomplete tetraplegia who developed $\mathrm{AD}$ complicated by a left parietal intracerebral hemorrhage and coma (Glasgow Coma Score 3), but eventually recovered. Comarr mentioned 4 fatal cases in the pre Guttmann/Whitteridge era ${ }^{16}$ diagnosed as hypertensive encephalopathy with seizures. It is very important to manage this syndrome energetically and the rule that prevention is better than cure has it best application in predisposed patients. Stimuli below the level of cord injury in such patients whether nociceptive and/or propriceptive should be avoided or promptly alleviated. Stimuli to the skin, mucosa of pelvic organs, smooth or striated muscles or even the bone should be looked for. Overdistension of a viscus (bladder, bowel or even stomach) may initiate a crisis. From the bladder, a plugged catheter, insertion of a catheter, irrigation or filling the bladder for cystometry, cystoscopy, cystography, urethral dilatation or other surgical procedures may precipitate AD. From the rectum, dyschesia, fecal impaction, digital 
evacuation, enemas, Barium roentgenography, rectal problems such as prolapsed hemorrhoids, fissures, perirectal suppuration, insertion of a rectal tube, ultrasound probe, can be precipitating stimuli. Sexual intercourse may lead to AD. In the extremities, ingrowing toenails, ungual suppuration, fractures, deep pressure sores, burns, etc, are to be looked for. Occasionally psychological stress is the underlying factor; its mechanism is not yet identified. In surgery, especially urological and rectal, surgery on pressure ulcers and in obstetric delivery one has to be alert for AD.

Preventive measures that we use are:

1 In predisposed patients before going to surgery or for a special procedure an oral capsule of $10 \mathrm{mgms}$ of Nifedipine is given 30 minutes before the procedure. We have also used Clonidine patch Number 1 or 2, 3 days before the procedure.

2 Spinal anesthesia may be used for urological rectal, pelvic or lower extremity surgery.

3 In patients with a spastic bladder, smooth muscle relaxants are routinely given (Banthine or Ditropan).

4 Susceptible patients are given alert cards explaining the condition and its management to retain in their wallets, especially for physicians who may not be familiar with AD.

The crisis may be over but status dysreflexicus must be kept in mind and the patient must be monitored for a least 24 hours. In our patient the crisis was over the first time the patient was catheterized and the patient was asleep when he developed the neurological changes. The attending physician and nurse should keep a close observation as the condition sometimes takes 'forme brusque'. Currently it is easy to diagnose intracranial pathology by CT scan or MRI and there should be no hesitation in making the diagnosis. In the early seventies (1974) the senior author had 2 patients who may be briefly mentioned. One was a 60 year old $\mathrm{T} 4$ paraplegic who was admitted in coma and had bowel impaction for 7 days. Lumbar puncture showed subarachnoid hemorrhage. The patient developed Cheyene-Stokes breathing and died. The second case was a 34 year old quadriplegic who was admitted for bowel impaction and developed cerebral hemorrhage leading to aphasia from which he eventually recovered.

It is known that nontraumatic intracranial hemorrhage is mostly a disease of the elderly and that its occurrence in patients less that 40 years of age accounts for only $3 \%$ of all stroke victims. In our population of approximately 3000 patients we have encountered 3 cases known to be secondary to autonomic dysreflexia, an incidence of $0.1 \%$ excluding all other causes of stroke. In Linden's series ${ }^{13}$ of 444 only one case died from $\mathrm{AD}$, an incidence of $-0.2 \%$. However, these figures are not statistically accurate since the causes of stroke in spinal cord injury are not well documented. From The National Spinal Cord Injury Data Bank 22 deaths out of 796 were due to CVA (DeVivo), ie $+/-2.7 \%$ without itemizing hemorrhage and thrombosis or embolism. In 1979 the death rate from cerebrovascular disease in the United States has declined from $66.3 / 100,000$ in 1970 to $42.5 / 100,000$ or $0.042 \%$. It seems therefore, that CVA has a higher incidence in the spinal cord injured and should be dealt with efficiently and the patients should be well monitored and, above all, preventive measures are most valuable.

\section{References}

1 Guttmann L (1976) Spinal Cord Injuries: Comprehensive Management and Research. Chapter 27. Blackwell Scientific Publications, London.

2 Comarr AE (1984) Autonomic dysreflexia (hyperreflexial). J Appl Physiol 7(3): 55-57.

3 Bors E, Comarr AE (1971) In: Karger S, editor. Neurological Urology. University Park Press, New York: 88.

4 Linden R, Leffler EJ, Kedia KR (1985) A comparison of the efficacy of an Alpha-I-adrenergic blocker and a slow calcium channel blocker in the control of autonomic dysreflexia. Paraplegia 23: 34-38.

5 Black J (1988) Autonomic dysreflexia/hyperreflexia in spinal cord injury: description and treatment. $A U A A$ J 8(4): 12.

6 Frankel HL, Mathias CJ (1979) Cardiovascular aspects of autonomic dysreflexia since Guttmann and 
Whitteridge (1947). Paraplegia 17: 46-51.

7 Gimovsky ML, Ojeda A, Ozaki R, Zerne S (1985) Management of autonomic hyperreflexia associated with low thoracic spinal cord lesion. Am J of Obstet Gynecol 153: 223-224.

8 Linden R, Leffler E, Freehafer A, Lyons AM, Hazel C (1986) Further Experience with Nifedipine in the Treatment of Autonomic Dysreflexia. Spinal Cord Injury Service, Cuyahoga County Hospital, Highland View, Cleveland, Ohio.

9 McGuire EJ, Rossier AB (1983) Treatment of acute autonomic dysreflexia. J Urol 129: 1185-1187.

10 Hilton $\mathrm{J}(1860)$ A course of lectures in pain and the therapeutic influences of mechanical and physiological rest in accidents and surgical diseases. Lancet October: 401-404.

11 Barnett HJM, Stein BM, Mohr JP, Yatsu FM (1986) Stroke Pathophysiology, Diagnosis, and Management. Volume I, Chapter 27. Churchill Livingstone, New York.

12 Yarkony GM, Katz RT, Wu YC (1986) Seizures secondary to autonomic dysreflexia. Arch Phys Med Rehabil 67: 834-835.

13 Linden R, Joiner E, Freehafer AA, Hazel C (1980) Incidence and clinical features of autonomic dysreflexia in patients with spinal cord injury. Paraplegia 18: 285-292.

14 Kursh ED, Freehafer A, Persky L (1977) Complications of autonomic dysreflexia. J Urol 118: 70-72.

15 Hanowell LH, Wilmot C (1988) Spinal cord injury leading to intracranial hemorrhage. Crit Care Med 16(9): 911-912.

16 Guttmann L, Whitteridge D (1947) Effects of bladder distension on autonomic mechanisms after spinal cord injuries. Brain 70: 361-405. 